\title{
White Paper on the Use of Team Calendars with the JIRA Issue Tracking System and Confluence Collaboration Tools for the XLPR Project \\ by
}

\author{
P. T. Williams, H. B. Klasky, and B. R. Bass
}

\section{Executive Summary}

ORNL was tasked by xLPR project management to propose a team calendar for use within the xLPR consortium. Among various options that were considered, the approach judged by ORNL to best fit the needs of the XLPR project is presented in this document. The Atlassian Team Calendars plug-in used with the Confluence collaboration tool was recommended for several reasons, including the advantage that it provides for a tight integration between Confluence (found at https://xlpr.ornl.gov/wiki ) and xLPR's JIRA ${ }^{1}$ issue tracking system (found at https://xlpr.ornl.gov/jira ).

This document is divided into two parts. The first part (Sections 1-6) consists of the white paper, which highlights some of the ways that Team Calendars can improve communication between xLPR project managers, group leads, and team members when JIRA is applied for both issue tracking and change-management activities. Specific points emphasized herein are as follows:

- The Team Calendar application greatly enhances the added value that the JIRA and Confluence tools bring to the XLPR Project.

- The Team Calendar can improve communication between xLPR project managers, group leads, and team members when JIRA is applied for both issue tracking and change-management activities.

- The Team Calendar works across different email tools such as Outlook 2011, Outlook 2010, Outlook 2007, Google Calendars and Mac's iCalendar to name a few.

- $\quad$ xLPR users can now access the wiki Confluence (with embedded Team Calendars) directly from JIRA without having to re-validate their login.

The second part consists of an Annex (Section 7), which describes how users can subscribe to Team Calendars from different calendar applications. Specific instructions are given in the Annex that describe how to

- Import xLPR Team Calendar to Outlook - Version Office 2010

- Import xLPR Team Calendar to Outlook - Version Office 2007

- Subscribe to Team Calendar from Google Calendar

The reader is directed to Section 4 for instructions on adding events to the Team Calendar or accessing ORNL staff for assistance with such additions. To seek help with your questions and problems regarding the content of this document, please contact Hilda Klasky at klaskyhb@ornl.gov

\footnotetext{
${ }^{1}$ To enable the JIRA integration features for Team Calendars, the Team Calendars for JIRA Integration Plugin must be installed in JIRA. This plugin is bundled in JIRA 4.4.x and later.
} 


\section{INTRODUCTION}

The use of the Atlassian Team Calendars plugin with the Confluence collaboration tool provides for a tighter integration between Confluence and XLPR's JIRA ${ }^{2}$ issue tracking system. JIRA Calendars show dates and deadlines for projects tracked in JIRA. Team Calendars enable project managers, group leads, and group members to

- communicate project and release plans to their team by visualizing issues, version, and custom due dates on a calendar that can be embedded anywhere in Confluence,

- understand how the availability of their team could impact their project's milestones by cross-referencing their team with personal calendars where group leaders can track team resource availability.

Team Calendars provides an integrated approach to mapping projects by offering JIRA savedfilter support. There are two use cases that make Team Calendars particularly useful for customers that use JIRA. Both use cases are relevant to the xLPR project. Figure 1 and Figure 2 below show scheduled events for the XLPR project during August and September 2012.

\section{Projects Using JIRA for Issue Tracking}

Using saved JIRA search filters, calendars can be created that track what's most important to the project. For one calendar, that might be "all open New Feature issues for a particular Fix Version". For another calendar, that might be "all open Bug issues for the current Fix Version". Using saved filters, only essential issues can be tracked. Combining saved JIRA filters with Team Calendars' support for custom issue due dates significantly enhances JIRA's issue tracking functionality.

\section{Projects Using JIRA for Change Management}

Viewing a list of issues derived from queries in JIRA's issue navigator is one way for project team members to visualize upcoming changes. Displaying scheduled changes on a calendar can significantly enhance this line of communication. Clear communication and visibility in any change management process is critical and the addition of saved JIRA filters provides an even better lens for project leaders and team members to stay on the same page. Team Calendars makes it easier to schedule change deployments and to communicate change, as well as quickly identify "what changed and when".

The following capabilities make the use of Team Calendars a worthwhile added value to aid xLPR with change management:

- Manage change schedules more effectively with JIRA search filter support.

- Communicate team workload - making it clear who's working which issue.

- Coordinate change across multiple systems - don't let complexity overwhelm the project by laying everything out clearly on a calendar.

\footnotetext{
${ }^{2}$ To enable the JIRA integration features for Team Calendars, the Team Calendars for JIRA Integration Plugin must be installed in JIRA. This plugin is bundled in JIRA 4.4.x and later.
} 
Page 3 of 11

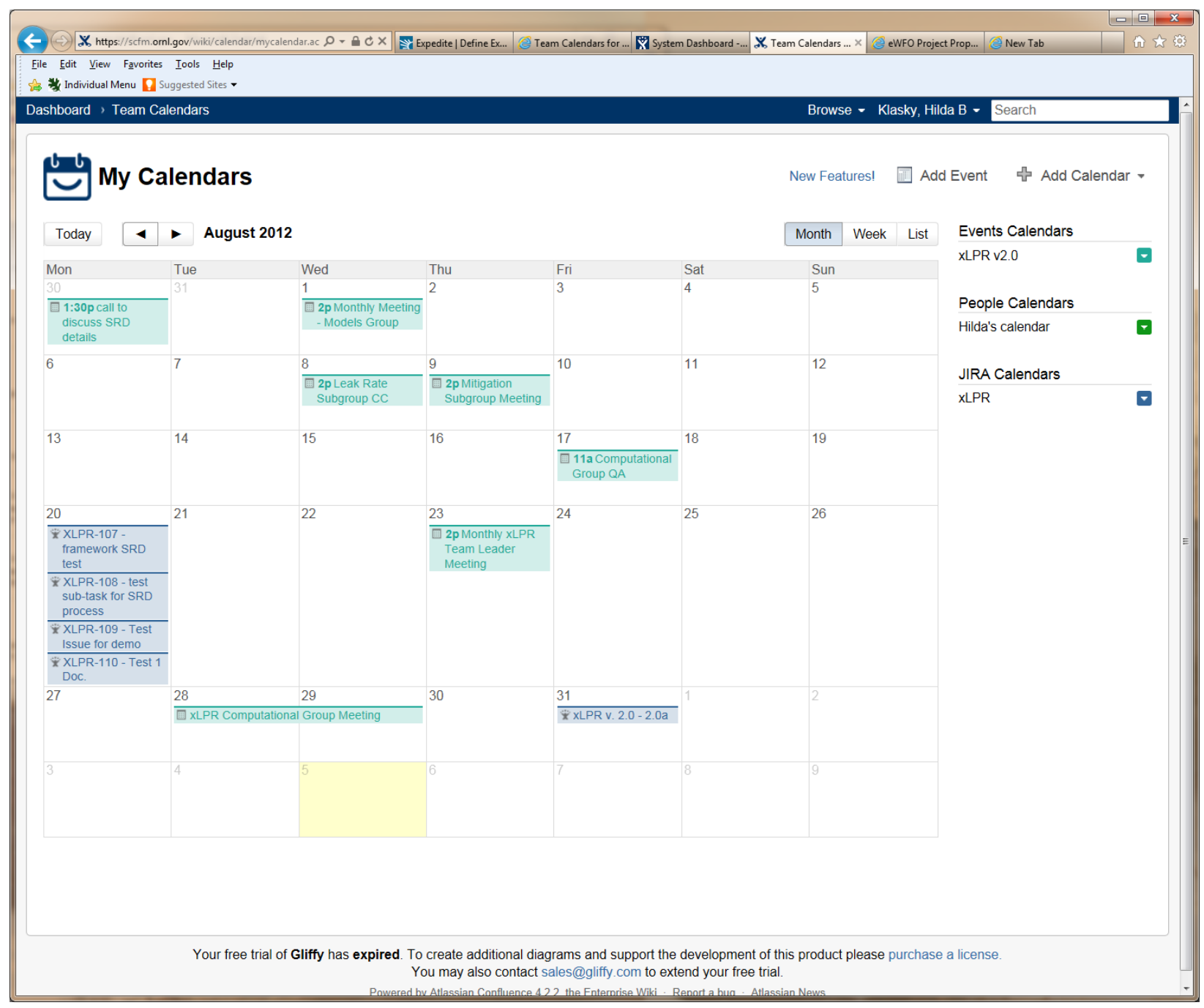

Figure 1 The xLPR Group Calendar August 2012 


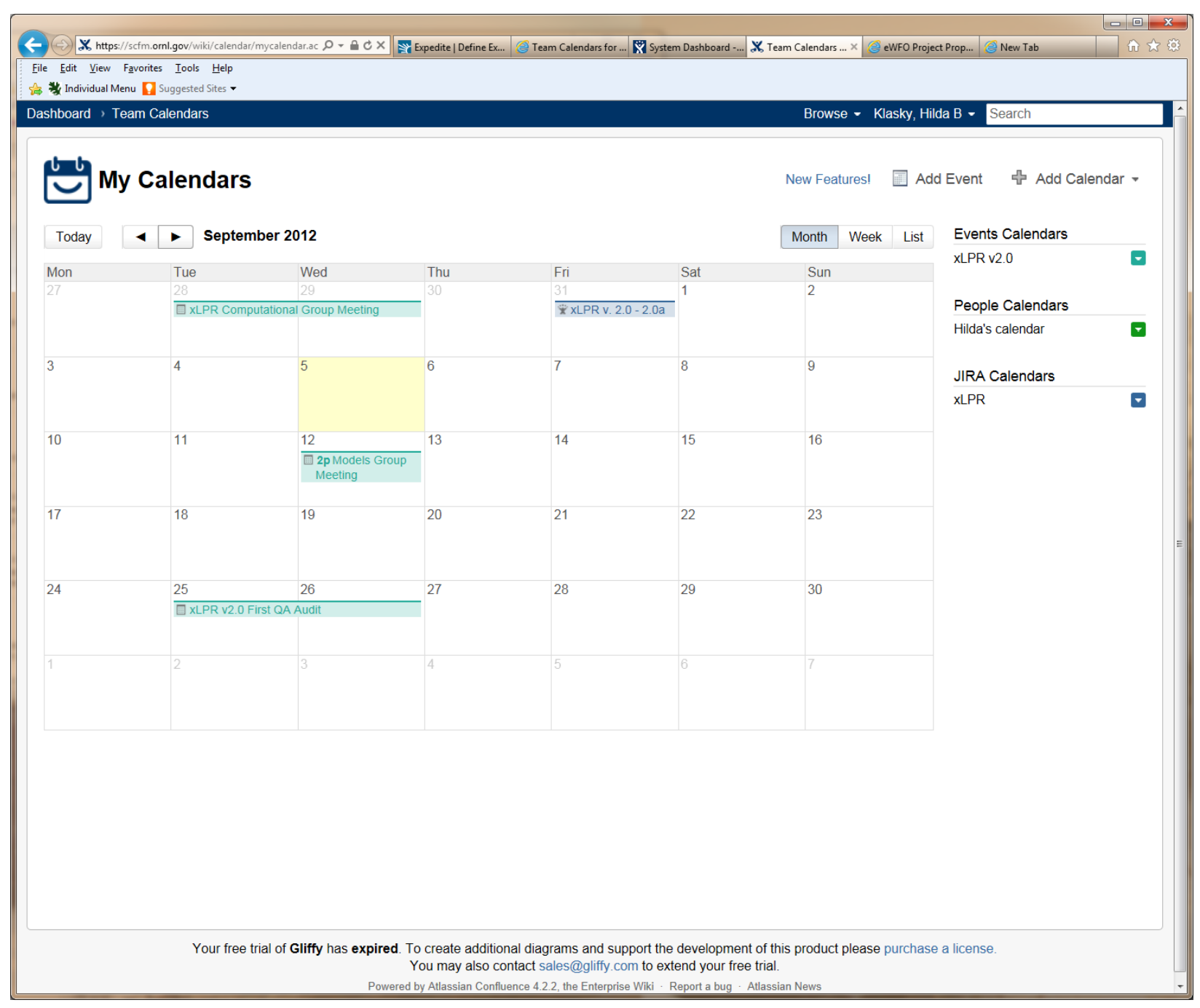

Figure 2 The xLPR Group Calendar September 2012

Moreover, to avoid the synchronization issues that SharePoint has, the Confluence Team Calendar is equipped with the following features:

1) Updating is done through the Confluence wiki.

2) Subscriptions can be done in Outlook and Google Calendar as a viewer mode.

The following features are available for closer integration between JIRA and Confluence:

\section{a) Fast Issue Creation}

Requirements developed in Confluence can be linked to actionable JIRA issues without leaving the Confluence editor. JIRA issues can be inserted in Confluence pages to generate reports that display key details - issue type, summary, and status. 


\section{b) Automatic Issue Linking}

Whenever a JIRA issue is mentioned in a Confluence page, JIRA will create an issue link to that page automatically. Issue links give you complete traceability from the requirements and specifications created in Confluence to the issues that are being tracked in JIRA.

\section{c) Centralize User Management}

If the user list is already set up in JIRA, there's no need to re-create them in Confluence. JIRA can be used as a central server for user management and authentication, and users can then access Confluence directly from JIRA without having to re-validate their login.

\section{Calendar Types}

You can have multiple calendars for different purposes in Confluence Team Calendar. There are, basically, two types of Calendars that can be shared among the team:

1. People Calendar: People calendars differ from events calendars by having a single person as the focus of each event. People calendars are ideally used for tracking things like annual leave dates, travel, and rosters.

2. Event Calendar: Events calendars differ from people calendars by having a location as well as a time and date. These are ideally used for things like dates for company group events, such as meetings.

\section{Adding Events to the Calendar is Easy}

Adding events to the calendar can be done by clicking 'Add Event' in all views, or by clicking on the calendar day in the month and week views. You'll be prompted to enter the title, time and other details. It will immediately appear on the calendar.

\subsection{Help adding xLPR events to the Team Calendar}

If you need help adding your meeting to the Team Calendar, ORNL staff provides the following options:

1) Email Angie Scott at scottar@ornl.gov to do it for you, also copy Hilda Klasky at klaskyhb@ornl.gov as backup. If possible, please inform of the meeting a week in advance as Angie works Monday to Wednesday only. You can always send an email to her. And include 'xlpr event' in the subject of the email, so Angie can identify your email with the xlpr project.

2) Include Angie Scott's email ( scottar@ornl.gov) in your distribution xlpr group/subgroup list. Please, include the project name: 'xlpr' in the subject of the email, so Angie can identify your invitation with the xlpr project.

3) Ask Hilda Klasky (klaskyhb@ornl.gov) if you need help and Angie Scott is not available to provide the requested help.

\section{Accessing the tool}

The xLPR Team Calendar can be accessed in the xlpr wiki home page at https://xlpr.ornl.gov/wiki/display/xLPR2/Home 


\section{Closure}

ORNL's task to provide a team calendar for the xLPR consortium has been presented in this white paper. Specific points emphasized here are given as follows:

a) The presented Team Calendar application greatly enhances the added value that the JIRA and Confluence tools bring to the XLPR Project.

b) The presented Team Calendar can improve communication between xLPR project managers, group leads, and team members when JIRA is applied for both issue tracking and change-management activities.

c) The presented xLPR Team Calendar works across different email tools such as Outlook 2010, Outlook 2007, Google Calendars and Mac's iCalendar to name a few.

d) XLPR users can now access the wiki Confluence (with embedded Team Calendars) directly from JIRA without having to re-validate their login.

e) ORNL staff (Angie Scott scottar@ornl.gov) can provide help to xLPR users to adding events into the xLPR Team Calendar.

To seek help with your questions and problems regarding the content of this white paper, please contact Hilda Klasky at klaskyhb@ornl.gov.

\section{Links for more information}

https://confluence.atlassian.com/display/TEAMCAL/Using+Team+Calendars

https://confluence.atlassian.com/display/TEAMCAL/Team+Calendars+2.4+Release+Notes

http://www.atlassian.com/software/team-calendars/whats-new/?tab=team-calendars-24

http://www.atlassian.com/software/team-calendars/overview

http://www.atlassian.com/software/confluence/overview/jira-users

http://www.ehow.com/how_2058421_add-internet-calendar-outlook-2007.html

https://confluence.atlassian.com/display/TEAMCAL/Subscribing+to+Team+Calendars+from+Go ogle+Calendar

https://confluence.atlassian.com/display/TEAMCAL/Subscribing+to+Team+Calendars+from+Mi crosoft+Outlook 
White Paper on Use of Team Calendars with JIRA/Confluence

Page 7 of 11

https://confluence.atlassian.com/display/TEAMCAL/Subscribing+to+Team+Calendars+from+Go ogle+Calendar 


\section{Annexes}

\subsection{Subscribing to Team Calendars}

Users can subscribe to Team Calendars from different calendar applications. Specific instructions are listed in the sections below.

Note: You cannot subscribe to JIRA Calendar types from Outlook

\subsection{Importing xLPR Team Calendar to Outlook (Office 2010)}

The following steps present how to import the xLPR Team Calendar to Outlook (Office 2010)

1. In Outlook, select "Open Calendar" > "From Internet". See Figure 3.

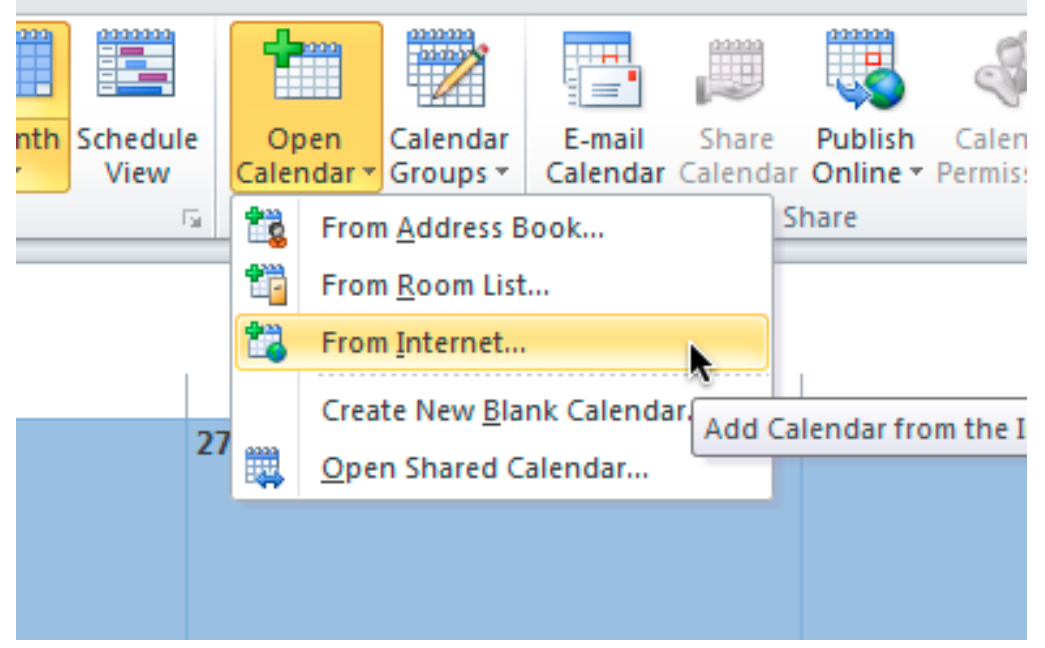

Figure 3 In Outlook, open calendar from Internet

2. Copy the following calendar address:

https://scfm.ornl.gov/wiki/rest/calendar-

services/1.0/calendar/export/subcalendar/private/1298c6342c8ec6458acd9f8d0598df0 d9a71a6d0.ics

3. Paste the calendar address as shown in Figure 4.

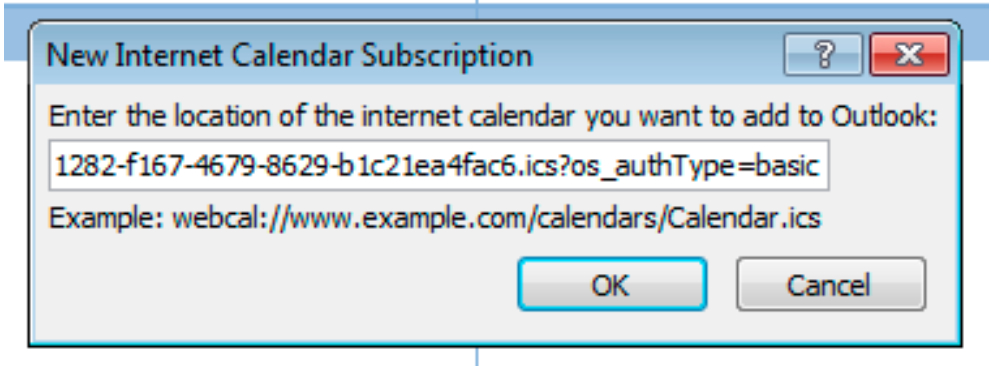

Figure 4 New Internet Calendar Subscription 
4. Configure the subscription. The name of the calendar file displays next to "Folder Name." Change it if necessary. Add a description to make the calendar easy to identify if you install additional calendars. Check whether you want attachments to download from the Internet calendar under "Attachments". Finally, select whether you want the subscription according to the publisher's recommendations. Checking this option ensures that you will not exceed the publisher's limitations and risk having your calendar account suspended. Click "OK" when finished.

5. Voila! Outlook will show the calendar. You will see both calendars side by side, and the calendar name has been added to the Other Calendars list. This side by side view is the default view, which can be modified. Figure 5 shows the xLPR Team Calendar in the overlay view in Outlook 2010.

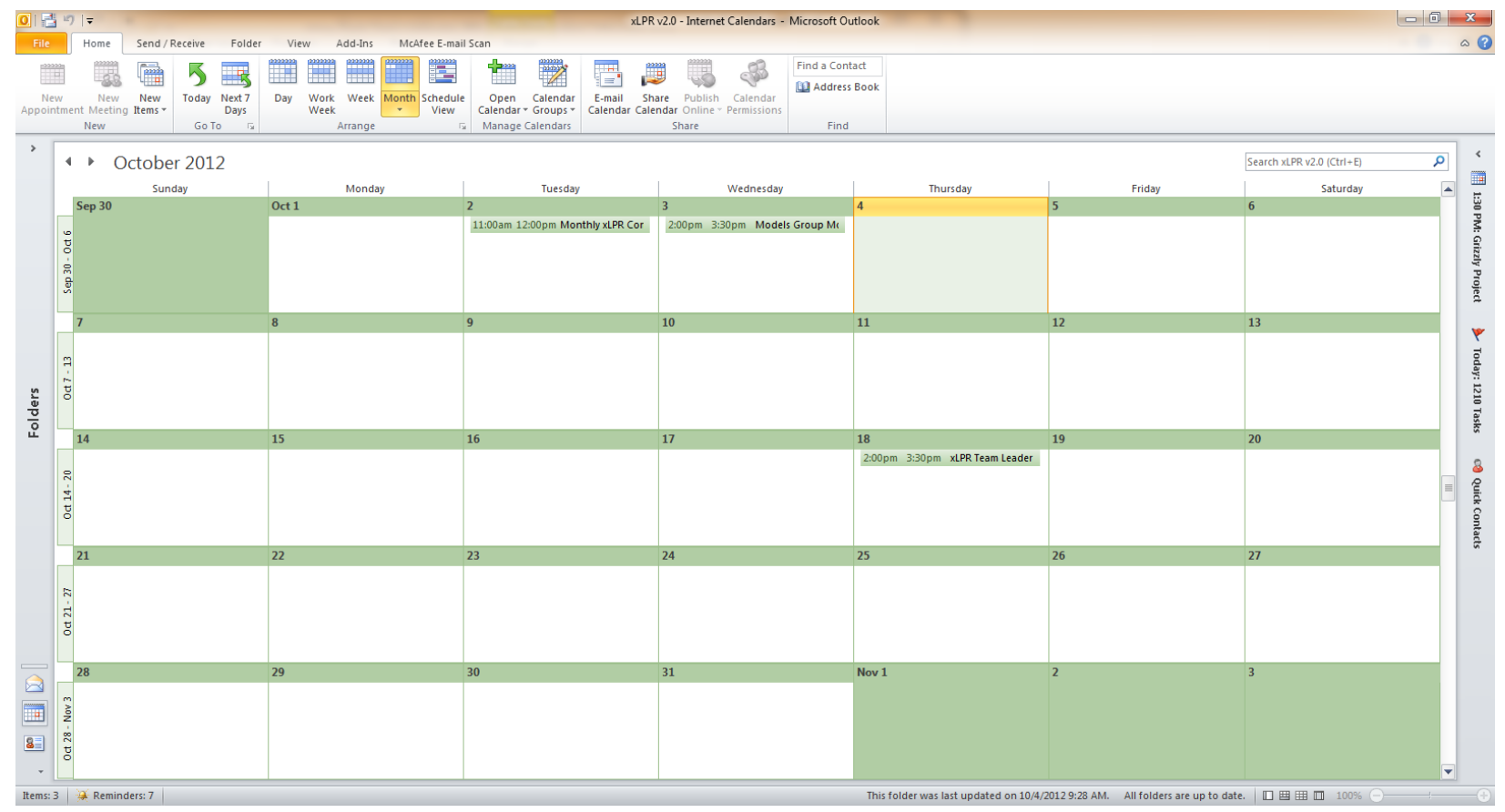

Figure 5 xLPR Team Calendar in Outlook

\subsection{Importing xLPR Team Calendar to Outlook (Office 2007)}

The following steps present how to import the xLPR Team Calendar to Outlook (Office 2007)

1. Start Outlook 2007. Make sure you have configured an active account.

2. Make sure any public sharing settings you need for remote calendar access are configured.

3. Insert the iCal link into Outlook 2007: In Outlook 2007, select "File," and then "Data File Management." Select the "Internet Calendars" tab. Click "New".

4. Copy the xLPR iCal URL: https://scfm.ornl.gov/wiki/rest/calendarservices/1.0/calendar/export/subcalendar/private/1298c6342c8ec6458acd9f8d0598 df0d9a71a6d0.ics 
And paste it into the URL field, and then hit "Add."

5. Configure the subscription. The name of the calendar file displays next to "Folder Name." Change it if necessary. Add a description to make the calendar easy to identify if you install additional calendars. Check whether you want attachments to download from the Internet calendar under "Attachments." Finally, select whether you want the subscription according to the publisher's recommendations. Checking this option ensures that you will not exceed the publisher's limitations and risk having your calendar account suspended. Click "OK" when finished.

6. Utilize your Internet Calendar. Click "Close" to return to Outlook 2007. After the Internet calendar is updated (may take several hours), it will display under "Other Calendars" in the Calendars section of Outlook 2007. Select it to manage your activities as you want them displayed on the Internet. You can use your Internet Calendar as you would internal Outlook 2007 calendars, such as displaying them side-by-side for easy copying of items from one to another.

Note: The XLPR Calendar in Outlook 2007 does not refresh in real time. Some XLPR users informed us that they need to close and re-open Outlook 2007 to refresh the xLPR Calendar.

\subsection{Subscribing to xLPR Team Calendar from Google Calendar}

\section{Setup}

1. Go to Google Calendar, select "Add by URL" from the "Other Calendars" menu as shown in Figure 6:

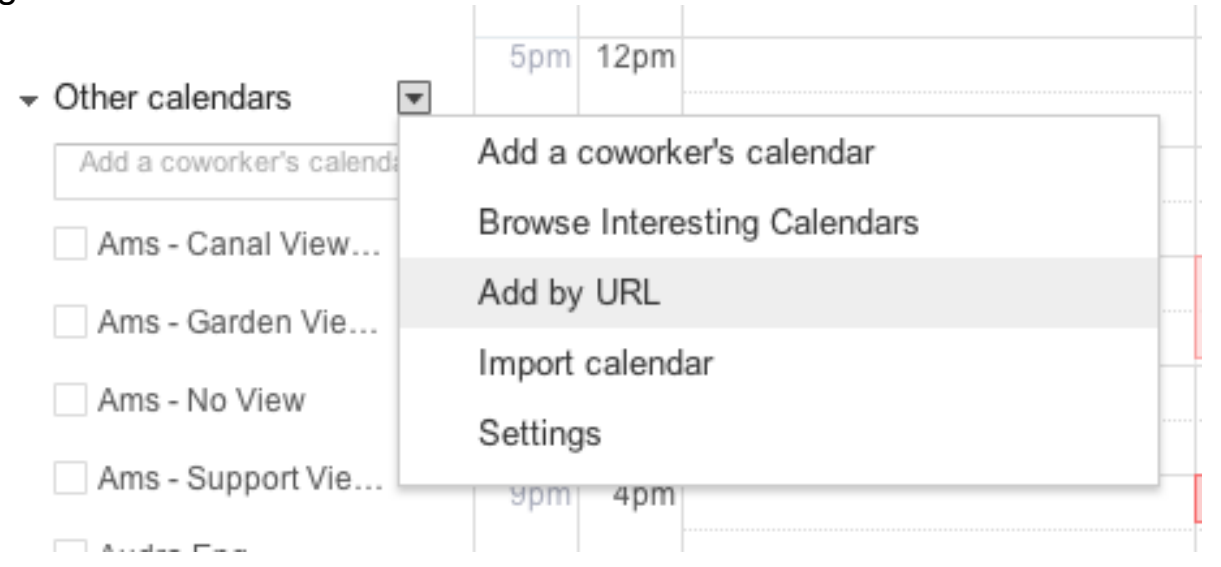

Figure 6 Google Add Calendar by URL

4. Paste the following XLPR Team Calendar URL:

\section{https://scfm.ornl.gov/wiki/rest/calendar-}

services/1.0/calendar/export/subcalendar/private/1298c6342c8ec6458acd9f8d0598df0 d9a71a6d0.ics

Users should be able to see the calendar XLPR Team Calendar shown in Figure 7 in Google calendar. 


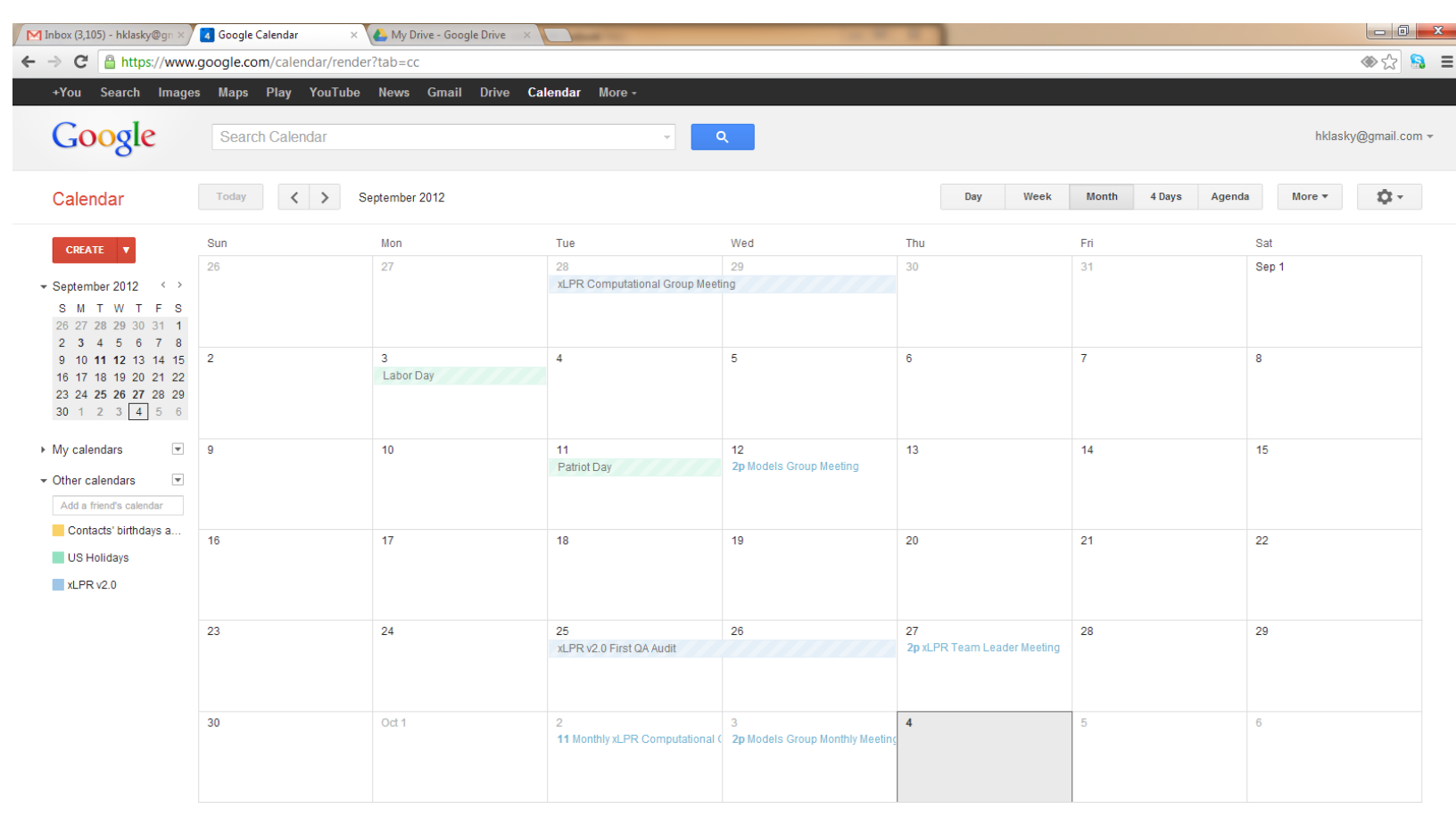

Figure 7 Google Calendar showing the xLPR Team Calendar

Note: Currently, calendar feeds are automatically updated by Google Calendar every few hours. The Google team understands that some users want to have the ability to refresh the calendars they added manually and they are exploring various ways to enable this. Thus, the XLPR Calendar in Google does not refresh in real time. 